\title{
Education Experiences of Adult Subjects and Caregivers for Mechanical Insufflation-Exsufflation at Home
}

\author{
Craig M Dale, Doug McKim, Reshma Amin, Sarah Carbone, Tom Fisher, Roger Goldstein, \\ Sherri Katz, Andrea Gershon, David Leasa, Mika Nonoyama, Regina Pizutti, Anu Tandon, \\ and Louise Rose
}

\begin{abstract}
BACKGROUND: In 2014, the Ministry of Health of Ontario, Canada, approved a program of public funding for specialist-prescribed mechanical insufflation-exsufflation (MI-E) devices for home use by individuals with neuromuscular respiratory insufficiency. Since 2014, 1,926 MI-E devices have been provided, exceeding device-use projections. Few studies describe the initial and ongoing education and support needs of home MI-E users and their family caregivers. This study aimed to explore the requirements of initial and ongoing education and support for MI-E device use, user confidence, and barriers and facilitators to home MI-E. METHODS: We conducted semi-structured interviews with new ( $<6$ months) and established (6-48 months) MI-E users and family caregivers. Device users rated their confidence on a numeric rating scale of 1 (not confident) to 10 (very confident). RESULTS: We recruited 14 new and 14 established MI-E users and caregivers (including 9 dyads), and we conducted 28 interviews. Both new and established users were highly confident in use of MI-E (mean \pm SD scores were $8.8 \pm 1.2$ and $8.3 \pm$ 2.1, respectively). Overall, the subjects were satisfied with their initial education, which consisted of a 1-2 h one-on-one session at home or in the clinic with a device demonstration and hands-on practice. Subjects viewed hands-on practice and teaching of caregivers as more beneficial than written materials. Ongoing support for device use was variable. Most subjects indicated a lack of specific follow-up, which resulted in uncertainty about whether they were using the MI-E device correctly or whether MI-E was effective. Facilitators to device utilization were ease of use, initial training, support from formal or informal caregivers, and symptom relief. Barriers were inadequate education on MI-E purpose, technique, and benefit; lack of follow-up; and inadequate knowledge of MI-E by nonspecialist health providers. CONCLUSIONS: The current model of home MI-E education at initiation meets user and caregiver needs. Better ongoing education and follow-up are needed to sustain the benefits through assessment of MI-E technique and its effectiveness. Key words: mechanical insufflation-exsufflation; neuromuscular disease; respiratory insufficiency; airway clearance; cough assist; self-management; patient education; teaching. [Respir Care 2020;65(12):1889-1896. (C) 2020 Daedalus Enterprises]
\end{abstract}

\section{Introduction}

For individuals living at home who develop respiratory insufficiency due to neuromuscular disorders, acute respiratory failure secondary to respiratory infection is the most

Dr Dale, Ms Carbone, and Dr Rose are affiliated with the Lawrence S. Bloomberg Faculty of Nursing, University of Toronto, Toronto, Canada. Dr Dale is affiliated with the TECC Program, Sunnybrook Health Sciences Centre and Research Institute, Toronto, Canada. Dr McKim is affiliated with the The Ottawa Hospital Respiratory Rehabilitation, The frequent reason for their unplanned access to health services, including emergency department visits and hospital admissions..$^{1-3}$ Progressive involvement of the respiratory muscles associated with neuromuscular disorders such as

Ottawa Hospital Sleep Centre and Research Institute, Ottawa, Canada. Dr McKim is affiliated with the Faculty of Medicine, University of Ottawa, Ottawa, Canada. Dr Amin is affiliated with the Division of Respiratory Medicine, SickKids Research Institute, University of Toronto, Toronto, Canada. Mr Fisher and Ms Pizzuti are affiliated with the Ventilator 


\section{EduCATION EXPERIENCES OF AdUltS ANd CAREgIVERS FOR MI-E}

muscular dystrophy, amyotrophic lateral sclerosis, and many others leads to recurrent atelectasis and pneumonia secondary to decreased cough efficacy as well as hypoventilation. ${ }^{4-9}$ Chronic respiratory failure is a frequent cause of death for these individuals.

Early and appropriate use of airway-clearance strategies such as mechanical insufflation-exsufflation (MI-E) combined with noninvasive ventilation can improve respiratory function ensuring fewer complications, reduce emergency department visits, and decrease acute care (including intensive care) admissions. ${ }^{10-14}$ It also decreases the need for tracheostomy and invasive ventilation, as well as their associated complications. MI-E therapy is a combination of invasive (ie, advanced airway) and noninvasive (ie, face mask) methods that clears respiratory secretions by applying positive pressure, followed by a rapid shift to negative pressure, thereby simulating a cough. ${ }^{10,11}$ Early and appropriate use of MI-E with noninvasive ventilation enables individuals with neuromuscular respiratory insufficiency to either remain at or return to home, thus minimizing health care utilization and costs, improving health-related quality of life, and extending life expectancy. ${ }^{1}$

On April 2014, the Ministry of Health and Long-term Care in Ontario, Canada, which serves a population of roughly 13 million citizens, approved the publicly funded Provincial Cough Assist Program. The prevalence of neuromuscular disease in this population is roughly 1,344 per 100,000 adults. ${ }^{1}$ The program, managed by the provincial Ventilator Equipment Pool, provides a publicly funded MI-E device (CoughAssist E70, Philips Respironics, Murrysville, Pennsylvania) for use in the home for individuals with neuromuscular respiratory insufficiency. ${ }^{15}$ Approved respiratory specialists prescribe the MI-E device to promote secretion clearance. Prior to April 2014, patients and their families purchased their own devices (or may have received private insurance coverage) at a cost of more than (CAD\$7,000) per device. As of June 2019 , the program has delivered $>1,926$ (on average 385 devices/y) devices to individuals living at home since its inception, well above the need predicted by the equipment pool. Although MI-E devices promote secretion clearance, thereby reducing the rate of respiratory infections and providing symptom relief, we hypothesized that

\footnotetext{
Equipment Pool, Kingston, Canada. Dr Goldstein is affiliated with the West Park Healthcare Centre, Toronto, Canada. Drs Goldstein, Gershon, and Tandon are affiliated with the Faculty of Medicine, University of Toronto, Toronto, Canada. Dr Katz is affiliated with the Children's Hospital of Eastern Ontario Research Institute, Ottawa, Canada. Dr Gershon is affiliated with the Department of Respirology and Clinical Immunology, Sunnybrook Health Sciences Centre and Research Institute, Toronto, Canada. Dr Gershon is affiliated with the Institute of Clinical Evaluative Sciences, Toronto, Canada. Dr Leasa is affiliated with the Department of Medicine, Divisions of Critical Care and Respirology, London Health Sciences Centre, London, Canada. Dr Leasa is affiliated
}

\section{QUICK LOOK}

\section{Current knowledge}

Mechanical insufflation-exsufflation (MI-E) devices promote secretion clearance and have the potential to reduce the rate of respiratory infections, emergency department visits, hospital admissions, and complications in patients with neuromuscular respiratory insufficiency. While education and support are required to promote adoption and adherence with prescribed MI-E use in the home, it is unknown if the current initial education and support are sufficient to meet the needs of MI-E users and their family caregivers.

\section{What this paper contributes to our knowledge}

Personalized one-on-one education sessions in clinic or the home with device demonstration and hands-on practice was key to high user confidence at initiation of MI-E use. However, improvements to ongoing user support are necessary. As cost savings derived from investment in health interventions such as MI-E are linked to the prevention of complications, clinic follow-up to reduce treatment uncertainties, improve understanding of treatment benefit, and promote device prescription adherence are warranted.

substantial initial and ongoing education and support are required to promote adoption and sustained adherence with prescribed MI-E use. ${ }^{10}$ It is unknown, however, whether the current initial and ongoing nonstandardized (ie, informal) patient education and support are sufficient to meet the needs of MI-E users and their family caregivers.

Therefore, through semistructured qualitative interviews, we sought to establish the user and family caregiver needs for initial and ongoing education and support for use of an MI-E device in the home, document confidence in MI-E device use, and establish barriers and facilitators to the use of home MI-E. An understanding of user and family caregiver needs as well as barriers and facilitators to MI-E use will inform approaches to promoting

with the Faculty of Medicine, Western University, London, Canada. Dr Nonoyama is affiliated with the Faculty of Health Sciences, Ontario Tech University, Oshawa, Canada. Dr Nonoyama is affiliated with the Rehabilitation Sciences Institute, University of Toronto, Toronto, Canada. Dr Tandon is affiliated with the Department of Respirology and Clinical Immunology, Sunnybrook Health Sciences Centre, Toronto, Canada. Dr Rose is affiliated with the Florence Nightingale Faculty of Nursing, Midwifery and Palliative Care, King's College London, London, United Kingdom. Dr Rose is affiliated with the Sunnybrook Research Institute, Toronto, Canada and also affiliated with the Institute of Clinical Evaluative Sciences, Toronto, Canada. 


\section{EdUCATION EXPERIENCES OF AdUlTS AND CAREgIVERS FOR MI-E}

device adherence, which will improve symptom management and reduce health care resource utilization for respiratory complications.

\section{Methods}

\section{Design}

We conducted a descriptive qualitative study using semistructured interviews from April 2016 to July 2017. Data were analyzed and interpreted using a social constructivist lens. This study was performed at the Ventilator Equipment Pool, Kingston, Canada, and at the University of Toronto, Toronto, Canada. This study was approved the research ethics boards of the University of Toronto and Kingston General Hospital. Participation was voluntary, and all subjects provided written consent prior to participation.

\section{Subjects}

Our eligibility criteria for MI-E users were: (1) they received an MI-E device through the Provincial Cough Assist Program; (2) they lived in a private dwelling; (3) they were able to speak English; (4) they were $\geq 12$ y old; (5) they were able to communicate via telephone; and (6) they consented to participate. Eligibility criteria for family caregivers were: (1) they were the family member or friend most responsible for providing or coordinating care without financial compensation; (2) they were able to speak English; (3) they were $\geq 18$ y old; and (4) they consented to participate. There were no exclusion criteria. MI-E users and family caregivers could be recruited as patient-caregiver dyads, although this was not a requirement for participation.

We recruited our sample from the Ventilator Equipment Pool database of MI-E users. We used a purposive sampling approach to recruit 14 MI-E users and 14 family caregiver subjects. ${ }^{16}$ User characteristics guiding recruitment included variation in sex, diagnostic category, age, and urban versus rural residence. Caregiver characteristics included sex, relationship to user (eg, spouse, parent, child), age of user,

Dr Rose presented a version of this study at the 2019 European Respiratory Society meeting, held September 28 to October 2, 2019, in Madrid, Spain.

This study was supported by a grant from the International Ventilator Users Network. The authors have disclosed no conflicts of interest.

Supplementary material related to this paper is available at http://www. rcjournal.com.

Correspondence: Craig M Dale RN PhD, Lawrence S. Bloomberg Faculty of Nursing, University of Toronto, 155 College St, Toronto, Ontario, Canada M5T1P8. E-mail: craig.dale@utoronto.ca.

DOI: $10.4187 /$ respcare.07534 diagnostic category of user, and urban versus rural residence. Seven of the MI-E users and 7 family caregivers had received the MI-E device within the previous 6 months and were included to explore initial education and support needs; 7 established MI-E users and 7 family caregivers using MI-E for $>6$ months were also included.

\section{Data Collection}

An experienced qualitative researcher on our team (CMD) conducted one-on-one telephone interviews using a semistructured interview guide. One interview was conducted with each subject, with no follow-up. Subjects were given the choice of completing the interviews individually or as a patient-caregiver dyads. The interview guide was sent to subjects ahead of time to enable reflection and preparation of responses. Patients and family caregivers were invited to rate their initial confidence using the MI-E on a numeric scale from 1 to 10 , with 1 being "not at all confident" and 10 being "very confident." Subjects were asked to reflect on facilitators and barriers to initial and ongoing MI-E use. Interviews were digitally audio-recorded and transcribed verbatim (see the supplementary materials at http://www.rcjournal.com).

\section{Data Analysis}

Differences between new and established MI-E users' selfreported confidence scores with using the MI-E device were analyzed using independent sample Student $t$ test/MannWhitney test in SPSS (version 23, IBM Armonk, New York). We analyzed interview transcripts using content analysis methods. ${ }^{17,18}$ We employed a deductive, 4 -step process to categorize and code facilitator and barrier statements from the interview texts. First, following an immersive reading of the transcripts, 2 members of the research team (CMD, SC) identified initial facilitator and barrier statements and patterns. Second, we iteratively created a code book with exemplar definitions. Third, the codes were applied across all interviews by one coder (SC). Fourth, we identified similarities and differences between subjects' accounts. The larger team was involved in the in-depth reading of the coding to ensure credibility. NVivo 11 software (QSR International, Doncaster, Australia) was used for all facets of the qualitative analysis. ${ }^{19}$

\section{Results}

Telephone interviews were undertaken with 28 subjects comprising 14 new and 14 established MI-E users and caregivers (including 9 dyads). Interviews took an average of 34 min to complete per subject or dyad. Eleven (79\%) MI-E users were male; mean age was 59.5 y (range 22-78). Ten $(71 \%)$ users required nocturnal bi-level positive airway pressure support or utilized a CPAP device; one user 


\section{EduCATION EXPERIENCES OF AdUltS ANd CAREgIVERS FOR MI-E}

Table 1. Subject Characteristics

\begin{tabular}{|c|c|c|}
\hline Characteristic & MI-E User & Caregiver \\
\hline New MI-E user ( $<6$ months) & $7(50)$ & $7(50)$ \\
\hline Established MI-E user ( $\geq 6$ months) & $7(50)$ & $7(50)$ \\
\hline Age, y & $59.5(22-79)$ & $56.9(35-70)$ \\
\hline \multicolumn{3}{|l|}{ Sex } \\
\hline Female & $3(21)$ & $12(86)$ \\
\hline Male & $11(79)$ & $2(14)$ \\
\hline \multicolumn{3}{|l|}{ Residence } \\
\hline Urban & $11(79)$ & $12(86)$ \\
\hline Rural (ie, population < 5,000) & $3(21)$ & $2(14)$ \\
\hline \multicolumn{3}{|l|}{ Primary diagnosis } \\
\hline Muscular dystrophy & $5(36)$ & NA \\
\hline Amyotrophic lateral sclerosis & $4(29)$ & NA \\
\hline Quadriplegia & $2(14)$ & NA \\
\hline Multiple sclerosis & $1(7)$ & NA \\
\hline Myasthenia gravis & $1(7)$ & NA \\
\hline Post-polio syndrome & $1(7)$ & NA \\
\hline \multicolumn{3}{|l|}{ MI-E application } \\
\hline Independent & $7(50)$ & NA \\
\hline Dependent/semidependent & $7(50)$ & NA \\
\hline \multicolumn{3}{|l|}{ Nocturnal ventilation } \\
\hline Bilevel positive airway pressure & $7(50)$ & NA \\
\hline CPAP & $3(21)$ & NA \\
\hline \multicolumn{3}{|l|}{ Continuous (24-h) ventilation } \\
\hline Assist control & $1(7)$ & NA \\
\hline $\begin{array}{l}\text { Data are presented as } n(\%) \text { or mean (range). } \\
\text { MI-E = mechanical insufflation-exsufflation } \\
\text { NA = not applicable }\end{array}$ & & \\
\hline
\end{tabular}

(7\%) required 24-h continuous mandatory ventilation via tracheostomy; 3 (21\%) users were not receiving home mechanical ventilation at the time of interview. Seven $(50 \%)$ users were fully or semidependent on caregivers to apply the MI-E device. Twelve (86\%) participating family caregivers were female; $10(71 \%)$ were spouses. Only one family caregiver had a health professional background (Table 1). Both new and established users were highly confident in use of MI-E; mean \pm SD scores were $8.8 \pm 1.2$ and $8.3 \pm 2.1$, respectively. Confidence scores were not significantly different between user groups $(P=.63)$.

We present the main results organized as facilitator themes and barrier themes, each with content focused on initial education needs for use of the MI-E device in the home; followup education and support needs to promote optimal use; perceptions of benefit; and ongoing use of the MI-E device. Each subject quote identifies the speaker role (eg, subject, family caregiver) and duration (eg, new, established) of MI-E use.

\section{Facilitators}

Initial Education Needs. Subjects identified considered oneon-one education sessions, either as individuals or dyadic (ie, patient and caregiver), that lasted $1-2 \mathrm{~h}$ with device demonstration and hands-on practice to be a successful strategy for initiating home use of MI-E. Patients endorsed a personalized and interactive approach to training led by a respiratory therapist (RT) educator in an out-patient clinic or at home, which included appropriate pacing, understandable information, and the opportunity to ask questions:

\section{"Not only did he show me, but he let me do it in front of him in case I had any questions.” [Established Patient User]}

Family and paid caregiver presence during initial training was perceived as essential for those physically dependent on others for their care needs. In addition, family presence was reported to positively influence recall of training content, including the purpose of MI-E, its component parts, settings, application technique, and cleaning instruction:

"I had my wife right there learning with me . . . in case I forget something, she knows." [New Patient User]

Twenty-two subjects (78\%) recommended provision of print or video resources to supplement one-on-one education sessions; however, less than half utilized such resources due to their stated preference for personalized instruction.

Follow-Up Education. Nineteen participants (68\%) recalled telephone follow-up by an RT educator in the weeks immediately following initial education. Both subjects and family caregivers described follow-up as a helpful reminder to use the device as instructed:

"So when [the educator] called me, it reminded me, 'Okay, I have to use it more.' So that's when I started to do it every morning." [New Patient User]

Follow-up was also noted as an opportunity to discuss early frustrations with MI-E use. For example, 6 subjects $(21 \%)$ described difficulty training home support workers who were not present at the time of the initial education:

"Well, I had some pretty good results right from the start. But learning how to get all the [home support workers] trained to hold the mask properly was one of the most difficult parts of it." [New Patient User]

Discussion with an RT educator was deemed helpful in surmounting early frustrations such as air leaks when using the mask interface, training home support workers to apply the device properly, and establishing a daily routine.

Perceptions of Benefit. Twenty-three subjects (82\%) perceived a benefit from the MI-E during introductory device instruction: 


\section{EdUCATION EXPERIENCES OF AdULTS AND CAREGIVERS FOR MI-E}

"I had the flu at that point. Like, I didn't really know how I was going to feel. But I thought, 'Wow this is really helping already!'” [New Patient User]

Six subjects $(21 \%)$ described a favorable sensation of their lungs "stretching" during the inhalation mode. Those using the device for $>6$ months reported benefit that included perceived maintenance or improvement in lung function:

"I definitely noticed that I've been maintaining what I'm at. That's for sure." [Established Patient User]

"We were always told he'd never regain his lung capacity, his diaphragm would never work again. [But the] cough assist has strengthened him. We could be wrong. A lot of doctors don't believe that that's what happened. We have a little bit of that belief." [Family Caregiver of Established Patient User]

Those experiencing respiratory infection during or following introduction to the MI-E reported an accelerated recovery through daily use:

"I had a cough and I actually woke up at night because I almost felt like I was suffocating. But then the next day, after using the machine, I mean it forced out a whole bunch of phlegm ... and the cold only lasted for 7 days. So [in the past when I] have a cold it lasts like 3 or 4 weeks." [Established Patient User]

Six experienced users $(21 \%)$ also perceived unanticipated reductions in health service utilization and antibiotic treatment for respiratory infection:

"[Patient] had a history of getting pneumonias for 4 years. And we've gone 6 months without, since he's had this [MI-E]. I can't believe it's just fluky." [Family Caregiver of Established Patient User]

"Oh I haven't been on an antibiotic, it's been 3 years now. I think that when you're able to clear your chest of the mucus and it's not sitting in your lungs, I think it helps." [Established Patient User]

Ongoing Use of the MI-E. Facilitators of ongoing MI-E use included ease of use, incorporation into preexisting routines, and reminders to use the device as prescribed. Twenty-four participants $(86 \%)$ reported the MI-E to be user-friendly:

"It's very easy to work the machine . . . because my settings are already in. So at nighttime when I use it, I just press the button and that fits for me. So it's quite simple and that is the way I wanted it to be really." [Established Patient User]
Participants reported the size of the device contributed to its flexible placement in the home where it could be incorporated into preexisting daily routines:

"We have it set up in the bedroom on that little table. It's one of the first things I do after I get him out of bed. I get him up, cleaned up, and dressed. And at the same time we use the cough assist machine just before we leave the bedroom." [Family Caregiver of New Patient User]

Patients with cognitive limitations reported that reminders for daily use were beneficial:

"My son put it into my phone to actually remind me. There's a little alarm that comes on at 10:00 in the morning and I think 4:00 in the afternoon and 9:00 or 10:00 at night reminding me to do my cough assist." [Established Patient User]

In summary, participants reported that personalized education, follow-up, perception of benefit, ease of use, and automated reminders facilitated initial and ongoing use of the MI-E at home (Table 2).

\section{Barriers}

Initial Education Needs. While most participants reported initial education to be sufficient, 2 users (7\%) reported insufficient instruction contributing to uncertainty regarding the best technique for using the MI-E.

\footnotetext{
"The physiotherapist or the RT showed me a little bit how to use it, set it up. But I don't really know how to run it. I've been increasing my pressure because I want to stretch my lungs more, but I don't think I'm using it quite as well as I should.” [New Patient User]
}

In these cases, gaps in educational content contributed to uncertainties about device use and benefits. Other reported educational gaps included methods for accessing mask interface options and replacement parts:

\footnotetext{
"I wanted to know from the Ventilation Equipment Pool, where did they get these things from? And they refer you back to your supplier. And when we talked to the Ventilation Pool, the woman there just said, well, she didn't know [the names of suppliers]. That's not good help." [Established Patient User]
}

Limited knowledge of methods for ordering device components contributed to delays in the replacement of required parts. Similarly, 4 subjects (14\%) reported uncertainties about optimal methods of cleaning the MI-E tubing and mask interface: 


\section{EduCATION EXPERIENCES OF AdUlTS AND CAREgIVERS FOR MI-E}

Table 2. Subject and Caregiver Recommendations for Initial and Ongoing MI-E Education

\begin{tabular}{|c|c|}
\hline Time Period & Recommendations \\
\hline Initial education & $\begin{array}{l}\text { Provide training and instructions to patients and caregivers one-on-one, offering device demonstrations and hands-on practice. } \\
\text { Offer users and caregivers print or video instructions on device use and maintenance. Instructions should include the following } \\
\text { topics: troubleshooting while using the device, cleaning the device, and obtaining replacement parts. }\end{array}$ \\
\hline Follow-up education & $\begin{array}{l}\text { Provide telephone or at-home outreach in the first few weeks following hospital discharge or device initiation. } \\
\text { Encourage users and caregivers to set reminders for themselves to ensure that they utilize the device on a regular basis. }\end{array}$ \\
\hline Continuing education & $\begin{array}{l}\text { Ensure adequate training on using the MI-E among hospital staff in case patients are admitted to acute care. } \\
\text { During follow-up visits, ensure that respiratory health professionals offer appraisal support (ie, positive feedback regarding } \\
\text { MI-E use). } \\
\text { Where possible, health professionals should download usage data to discuss with clients. }\end{array}$ \\
\hline
\end{tabular}

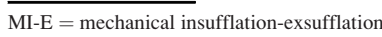

"My concerns are keeping it clean. I didn't find instructions on how to do that well enough. Right? And I'm just hoping I'm doing it well enough." [New Patient User]

Family caregivers also reported a need for better instruction on cleaning the mask interface and tubing to prevent infection.

Follow-up Education. Although 19 subjects (68\%) recollected an initial telephone follow-up, they recommended intermittent ongoing follow-up as a way to detect and resolve treatment difficulties, especially for those experiencing changes in their cognitive or physical health:

\footnotetext{
"Especially for senior people who are using it. Because sometimes it seems very clear at the time and then, you know, a couple of days later it might be 'Oh, what did they tell me?"' [Established Patient User]

"I was not thinking about the future as he loses the strength in his arms as to what to do. So there was no education on that." [Family Caregiver of New Patient User]
}

Six subjects $(21 \%)$ reporting infrequent interaction with respiratory health providers described unresolved treatment difficulty:

\footnotetext{
"We have one problem that's never been resolved for us; bottom line, the face mask. The fitting of the face mask is still difficult, and we don't seem to get a real good seal." [New Patient User]
}

Ongoing contact with RT educators was described as a welcome opportunity for clinical support and the collaborative resolution of impediments to prescribed MI-E use.

Perceptions of Benefit. Subjects using the device for several months wanted to know about the impact of MI-E use on their respiratory function. However, 18 subjects $(64 \%)$ could not recall any discussion of the beneficial impact of MI-E on their respiratory health when returning to the original prescriber. In one case, a subject who had been prescribed MI-E during acute hospitalization had not been referred to a pulmonologist for follow-up measures of respiratory function:
"We were hoping to possibly get a referral to a respirolo- gist because, you know, we're interested to see how much better his lungs have expanded. You know, he's been home over a year and yet there's been no call for follow- ups." [Family Caregiver of Established Patient User]

Although subjects were informed that MI-E usage records could be digitally downloaded from the device for expert evaluation and feedback, few described this occurring:

\footnotetext{
"No, there's never been any feedback about, you know, what they see on the memory card." [Established Patient User]

"You know, I don't even know what the results tell them. So if it's something that would benefit how we use it then totally that would be helpful." [Family Caregiver of New Patient User]
}

Lack of prescriber follow-up on MI-E usage and its effectiveness made subjects uncertain of proficient home application and the health benefits of adherence.

Ongoing Use of MI-E. Reports of difficulty with MI-E were primarily during its early use. Users employing an older model reported limitations in travel due to the device size, weight, and lack of built-in battery. In addition, the older model included a dial interface that could be unintentionally adjusted, thereby leading to inappropriate pressure delivery:

"I don't bring the machine with me, it's very heavy and awkward to move. It's one of the older ones, it's not the small electronic ones with a built in battery. So there's dials on it, and it is very hard to get it to the exact settings ... we found that the settings easily get changed, so 


\section{EdUCATION EXPERIENCES OF AdUlTS AND CAREGIVERS FOR MI-E}

we've got them written down so we can put them back on." [Family Caregiver of Established Patient User]

Others reported challenges with ongoing MI-E use when hospitalized. For example, two subjects encountered variable knowledge and skill among hospital clinicians that impeded MI-E use:

"I ended up spending some time in a hospital and in the rehab center. And we did bring my Cough Assist because I wanted to use it and all that. And there wasn't much of the staff at the hospital who were familiar with the machine. We had to train them on it. They were all like, 'Oh, what's this?"” [Established Patient User]

In summary, insufficient education regarding MI-E purpose, technique, and maintenance were reported as key barriers to ongoing use. Perceived limitations to travel and gaps in hospital-based clinician skill and knowledge could impede continuity of MI-E treatment. Finally, lack of prescriber follow-up made subjects uncertain of MI-E benefit and the requirement for treatment adherence.

\section{Discussion}

In this qualitative interview study of new and experienced MI-E users and their family caregivers, subjects' perspectives suggest that the existing model of initial education to establish MI-E home use meets most subjects' needs and creates user confidence. However, improvements to ongoing support are required to mitigate potential treatment nonadherence, which is a significant problem in individuals prescribed home ventilation technologies. ${ }^{20}$ Facilitators of device use included personalized one-on-one education, telephone follow-up, patient/family perception of benefit, ease of use, and automated treatment reminders. Barriers to device use included insufficient education regarding MI-E purpose, technique, and maintenance. Device characteristics, such as its weight, impeded the mobility of some subjects. Moreover, limited prescriber follow-up and feedback inhibited understanding of the benefits of MI-E treatment that may influence treatment adherence.

Important findings from this study include the positive impact on user confidence of personalized one-on-one education sessions in the clinic or at home with device demonstration and hands-on practice. Consistent with other research, subjects emphasized the importance of practical training that included thorough instruction on handling and maintaining the device for successful use. ${ }^{12}$ A personalized and interactive approach, with training and supportive presence of family caregivers and paid home support workers, was also perceived as key to successful establishment of device use and high user confidence. ${ }^{21}$ Our results align with studies indicating improvements in patient chronic disease knowledge, treatment adherence, and self-efficacy through the teach-back method (ie, return demonstration). ${ }^{22,23}$ Personalized education with follow-up may contrast with other patient encounters with health professionals in that time pressures can result in patients feeling uncared for, too intimidated to ask questions, and ultimately unprepared to care for themselves. ${ }^{23}$

Patient and health system benefits derived from investment in MI-E devices and their support include the prevention of complications leading to hospitalization. ${ }^{24} \mathrm{We}$ observed that positive user perception of MI-E treatment benefit included a reduction in the frequency and duration of respiratory illness, health service utilization, and antibiotic prescription. Positive perceptions of benefit may help to maintain user adherence required for the prevention of complications. Prior research suggests that the quality of life of individuals experiencing chronic illness improves when they demonstrate capacity in managing their own care. ${ }^{25}$ Self-efficacy or confidence to carry out a behavior may be enhanced through ongoing appraisal support (ie, feedback about their knowledge, skill, and outcomes related to prescribed treatment). Supportive dialogue with providers may help patients reduce treatment uncertainties, improve understanding of treatment benefit including the prevention of complications, and promote device prescription adherence. ${ }^{26}$

Barriers to MI-E use identified in this study may be resolved through proactive clinic follow-up to enable feedback on appropriate device use and benefit. Our subjects reported infrequent interaction with respiratory health professionals, including the MI-E prescriber. Intermittent clinic or home follow-up may reinforce prior learning and aid resolution of unexpected contingencies with device use. Previous research investigating transitions to home mechanical ventilation similarly reported patient preference for personalized and ongoing support from respiratory health professionals. ${ }^{27}$ Proactive RT educator follow-up detailing an individualized MI-E prescription, user technique, device cleaning, and methods for obtaining replacement parts is supported by growing evidence suggesting that patients with chronic conditions do better with selfmanagement through regular follow-up ${ }^{28}$ comprising educational, supportive, or behavioral interventions. ${ }^{20}$

Strengths of this study are the inclusion of new and established MI-E users and family caregivers representing variations in age, primary diagnosis, and treatment independence. Our patient-oriented focus on barriers and facilitators to the establishment of the MI-E was useful for identifying modifiable contributors to successful patient education and experiences. These results may not be generalizable to users in jurisdictions without a similar model of Ventilator Equipment Pool support. Self-reported confidence scores may have been influenced by response bias. 


\section{EdUCATION EXPERIENCES OF AdUltS AND CAREGIVERS FOR MI-E}

\section{Conclusions}

In this qualitative study of education and support needs for new and established MI-E users and their caregivers, we observed that the current model of one-on-one education with demonstration and hands-on practice with the device in the home or clinic at initiation met user and caregiver needs. User perception of MI-E treatment benefit included a reduction in the frequency of respiratory illness and health service utilization. Improvements to user support, including proactive clinic and home follow-up, however, are needed to sustain benefit due to a lack of ongoing assessment of MI-E technique and MI-E effectiveness. This model of equipment provision and clinical support could therefore be improved upon and adopted for use in other regions or jurisdictions to support continued MI-E use in the home and to benefit individuals with neuromuscular disease and respiratory insufficiency.

\section{REFERENCES}

1. Rose L, McKim D, Leasa D, Nonoyama M, Tandon A, Bai YQ, et al. Trends in incidence, prevalence, and mortality of neuromuscular disease in Ontario, Canada: a population-based retrospective cohort study (2003-2014). PLoS One 2019;14(3):e0210574.

2. Auger C, Hernando V, Galmiche H. Use of mechanical insufflationexsufflation devices for airway clearance in subjects with neuromuscular disease. Respir Care 2017;62(2):236-245.

3. Morrow B, Zampoli M, van Aswegen H, Argent A. Mechanical insufflation-exsufflation for people with neuromuscular disorders. Cochrane Database Syst Rev 2013;12:CD010044.

4. Benditt JO, Boitano LJ. Pulmonary issues in patients with chronic neuromuscular disease. Am J Respir Crit Care Med 2013;187(10):10461055.

5. Baydur A. Long-term noninvasive ventilation in the community for patients with musculoskeletal disorders: 46 year experience and review. Thorax 2000;55(1):4-11.

6. Tzeng A, Bach J. Prevention of pulmonary morbidity for patients with neuromuscular disease. Chest 2000;118(5):1390-1396.

7. Birnkrant DJ, Bushby K, Bann CM, Alman BA, Apkon SD, Blackwell A, et al. Diagnosis and management of Duchenne muscular dystrophy, part 2: respiratory, cardiac, bone health, and orothopaedic management. Lancet Neurol 2018;17(4):347-361.

8. Chatwin M, Ross E, Hart N, Nickol AH, Polkey MI, Simonds AK, et al. Cough augmentation with mechanical insufflation/exsufflation in patients with neuromuscular weakness. Eur Respir J 2003;21(3):502508.

9. McKim D, Katz S, Barrowman N, Ni A, LeBlanc C. Lung volume recruitment slows pulmonary function decline in Duchenne muscular dystrophy. Arch Phys Med Rehabil 2012;93(7):1117-1122.

10. Gomez-Merino E, Bach J. Duchenne muscular dystrophy: prolongation of life by noninvasive ventilation and mechanically assisted coughing. Am J Phys Med Rehabil 2002;81(6):411-415.
11. Chatwin M, Toussaint M, Gonçalves MR, Sheers N, Mellies U, Gonzales-Bermejo J, et al. Airway clearance techniques in neuromuscular disorders: a state of the art review. Respir Med 2018;136:98-110.

12. Siewers V, Holmøy T, Frich JC. Experiences with using mechanical in-exsufflation in amyotrophic lateral sclerosis. Eur $\mathrm{J}$ Physiother 2013; 14:201-207.

13. Sheers N, Howard M, Berlowitz D. Respiratory adjuncts to NIV in neuromuscular disease. Respirology 2019;24(6):512-520.

14. Rose L, McKim DA, Katz SL, Leasa D, Nonoyama M, Pedersen C, et al. Home mechanical ventilation in Canada: a national survey. Respir Care 2015;60(5):695-704.

15. Pereira J. New provincial ventilator service available through $\mathrm{KGH}$ Cough-assist device to improve life for patients with breathing problems. KGH Connect 2014 Mar 31http://www.kingstonhsc.ca/kghconnect/ news/new-provincial-ventilator-service-available-through-kgh Accessed June 20, 2019.

16. Patton M. Purposeful sampling. Qualitative evaluation and research methods. Beverly Hills, California: Sage Publications; 1990:169-186.

17. Bengtsson M. How to plan and perform a qualitative study using content analysis. NursingPlus Open 2016;2:8-14.

18. Vaismoradi M, Turunen H, Bondas T. Content analysis and thematic analysis: implications for conducting a qualitative descriptive study. Nurs Health Sci 2013;15(3):398-405.

19. Richards L. Handling qualitative data: a practical guide. London, UK: Sage Publications; 2005.

20. Wozniak DR, Lasserson TJ, Smith I. Educational, supportive and behavioural interventions to improve usage of continuous positive airway pressure machines in adults with obstructive sleep apnoea. Cochrane Database Syst Rev 2014;1:CD007736.

21. Bamm EL, Rosenbaum P. Family-centered theory: origins, development, barriers, and supports to implementation in rehabilitation medicine. Arch Phys Med Rehabil 2008;89(8):1618-1624.

22. Ha Dinh TT, Bonner A, Clark R, Ramsbotham J, Hines S. The effectiveness of the teach-back method on adherence and self-management in health education for people with chronic disease: a systematic review. JBI Database System Rev Implement Rep 2016;14(1):210247.

23. Jack BW, Chetty VK, Anthony D, Greenwald JL, Sanchez GM, Johnson $\mathrm{AE}$, et al. A reengineered hospital discharge program to decrease rehospitalization: a randomized trial. Ann Intern Med 2009; 150(3): 178-187.

24. Vitacca M, Paneroni M, Trainini D, Bianchi L, Assoni G, Saleri M, et al. At home and on demand mechanical cough assistance program for patients with amyotrophic lateral sclerosis. Am J Phys Med Rehabil 2010;89(5):401-406.

25. Briscoe WP, Woodgate RL. Sustaining self: the lived experience of transition to long-term ventilation. Qual Health Res 2010;20(1):57-67.

26. King J, O’Neill B, Ramsay P, Linden MA, Darweish Medniuk A, Outtrim J, Blackwood B. Identifying patients' support needs following critical illness: a scoping review of the qualitative literature. Crit Care 2019;23(1): 187

27. Dale CM, King J, Nonoyama M, Carbone S, McKim D, Road J, Rose L, CANuVENT group. Transitions to home mechanical ventilation: the experiences of Canadian ventilator-assisted adults and their family caregivers. Ann Am Thorac Soc 2018;15(3):357-364.

28. Epping-Jordan JE, Pruitt SD, Bengoa R. Improving the quality of health care for chronic conditions. Qual Saf Health Care 2004;13 (4):299-305. 